\title{
Improved electrical properties of wafer-bonded $p$-GaAs/n-InP interfaces with sulfide passivation
}

\author{
Keisuke Nakayama, ${ }^{\text {a) }}$ Katsuaki Tanabe, and Harry A. Atwater ${ }^{\text {b) }}$ \\ Thomas J. Watson Laboratory of Applied Physics, \\ California Institute of Technology, Pasadena, California 91125, USA
}

(Received 10 December 2007; accepted 27 February 2008; published online 2 May 2008)

\begin{abstract}
Sulfide-passivated $\mathrm{GaAs}$ and $\mathrm{InP}$ wafers were directly bonded to explore the efficiency of sulfide passivation on the bonded interfacial properties. We find that the bonded GaAs/InP interfaces after sulfide passivation contain sulfur atoms and a decreased amount of oxide species relative to the pairs bonded after conventional acid treatment; however, the residual sulfur atoms have no effect on the bonding strength. The electrical properties of the bonded $p$-GaAs $/ n$-InP heterojunctions were studied for different acceptor concentrations in $p$-GaAs. A reduced interfacial trap state density enhances the tunnel current flow across the depletion layer in the sulfide-passivated case. A directly bonded tunnel diode with a heavily doped $p$-GaAs $/ n$-InP heterojunction was achieved when the wafers were sulfide passivated and then bonded at temperatures as low as $300{ }^{\circ} \mathrm{C}$. This sulfide-passivated tunnel diode can be used for fabrication of lattice-mismatched multijunction solar cells in which subcells are integrated via direct bonding. () 2008 American Institute of Physics.

[DOI: $10.1063 / 1.2912717$ ]
\end{abstract}

\section{INTRODUCTION}

III-V multijunction solar cells, which consist of subcells with different energy band gap materials, enable increased conversion efficiency due to the higher absorption efficiency obtained from spectrum splitting. ${ }^{1}$ For the high efficiency performance of monolithically fabricated multijunction solar cells, high quality epitaxial growth of subcells with a high internal quantum efficiency is a requirement as well as an optimized combination of band gap materials for efficient conversion of the solar spectrum. ${ }^{2-5}$ While metamorphic three junction cells, in which active cells are grown lattice mismatched to the growth substrate via compositionally graded buffer layers, result in a high efficiency of over $40 \%$ at 240 suns, ${ }^{5}$ preparation of a higher-performance multijunction solar cell with four or more band gaps will need more design freedom and inherently higher quality active regions of subcells.

An alternative approach is to use direct wafer bonding to interconnect between subcells in multijunction solar cells. ${ }^{6,7}$ This technique enables further flexibility in achieving an optimal band gap sequence for solar energy conversion and fabrication of dislocation-free active cells by confining the lattice-mismatch accommodation to the bonded interfaces. We have focused on the fabrication of a multijunction solar cell wherein high band gap GaAs lattice-matched subcells (InGaP, GaAs) are combined to lower band gap InP latticematched subcells (InGaAsP, InGaAs) via direct wafer bonding. ${ }^{8}$ In order to monolithically interconnect between the top and bottom subcells, the bonded InP/GaAs heterojunction must be a highly conductive Ohmic junction. The electrical properties of both $n$-GaAs $/ n$-InP and

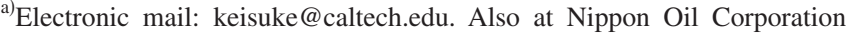
Yokohama, Japan 231-0815.

b)Electronic mail: haa@caltech.edu.
}

$p$-GaAs/p-InP bonded heterojunctions have been investigated and analyzed based on thermionic emission models and tunnel effect across the energy barriers. The interfacial conductivity of $n$ - $n$ and $p$ - $p$ heterojunctions was found to be improved by an increase in doping concentrations, bonding temperature, and removal of oxygen at the interfaces. ${ }^{9-11} \mathrm{We}$ previously fabricated a GaAs/InGaAs tandem solar cell by utilizing a direct bonding of $n$-GaAs $/ n$-InP. ${ }^{11}$ In this structure, a heavily doped layer for the tunnel diode was grown on the GaAs structure to switch polarity for the bonded interface. The additional layer reduces the incident light intensity because of the free carrier absorption. A wafer-bonded tunnel diode consisting of a heavily doped $p$-GaAs $/ n$-InP heterojunction, as shown in Fig. 1, provides a direct way to interconnect between the subcells in series without any additional layers. However, the electrical characteristics of bonded $p$-GaAs $/ n$-InP wafers have not been reported thus far, while heavily doped $p$-Si/n-Si tunnel diodes were successfully demonstrated by wafer bonding. ${ }^{12}$

In this study, we fabricated $p$-GaAs/ $n$-InP heterojunctions via direct wafer bonding and we first investigated the

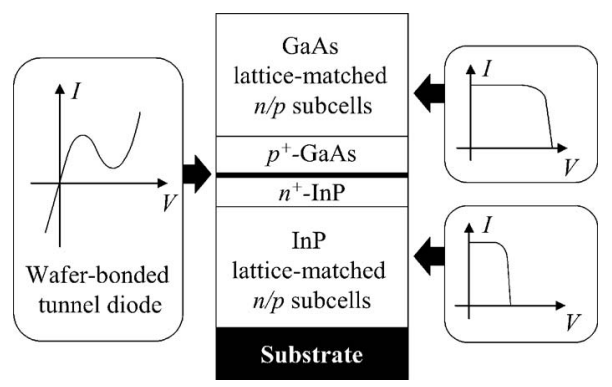

FIG. 1. The structure of a multijunction solar cell via direct wafer bonding, with characteristics of the upper subcells lattice matched to GaAs, the lower subcells lattice matched to InP, and interconnection between the subcells by wafer-bonded tunnel diode. 
effect of sulfide passivation of the bonded surfaces on the electrical properties of the heterojunctions. Surface treatments for III-V compound semiconductor wafers by sulfide compounds were shown to enhance the photoluminescence response and improve the electrical properties by removing the oxygen on the surfaces and reducing the surface trap state densities. ${ }^{13-18}$ Although this well-known technique has never been employed for direct bonded interfaces, this study shows that the wafers with residual sulfur atoms can be directly bonded without decreasing the bonding strength at the interfaces. In addition, sulfide-passivated interfaces give a significant improvement of the interfacial electrical conductivities and direct bonding of heavily doped $p$-GaAs and $n$-InP wafers after sulfide passivation forms a tunnel diode at temperatures as low as $300{ }^{\circ} \mathrm{C}$. Sulfide passivation induces the tunnel current flow across the depletion region of the $p$-GaAs $/ n$-InP heterojunction by reducing the interfacial trap state densities, which increases the built-in potential and, thus, the electric field at the bonded interface.

\section{EXPERIMENT}

350- $\mu$ m-thick epiready (100) GaAs and (100) InP wafers with various doping concentrations were used for direct wafer bonding experiments. The cleaved wafers were cleaned with a Va-Tran $\mathrm{CO}_{2}$ Sno-Gun II, followed by chemical etching in 4:1:1 $\mathrm{H}_{2} \mathrm{SO}_{4}(98 \%): \mathrm{H}_{2} \mathrm{O}_{2}(30 \%): \mathrm{H}_{2} \mathrm{O}$ solutions for $1 \mathrm{~min}$. The sulfide passivation of the wafers was achieved by dipping in 1:9 ammonium sulfide (20\%): tertbutanol solutions for $30 \mathrm{~s}$ and then rinsing in de-ionized water and drying with $\mathrm{N}_{2}$. Both the GaAs and the InP surfaces were hydrophilic after sulfide passivation. Then, the wafers were brought into contact and annealed at $1 \mathrm{MPa}$ at temperatures of $200-400{ }^{\circ} \mathrm{C}$ in atmosphere for $3 \mathrm{~h}$ and cooled down to room temperature, denoted as "S pairs." Reference bonded samples, denoted as "A pairs," were prepared with a similar procedure but with a dip in $1: 3 \mathrm{HCl}$ $(35 \%): \mathrm{H}_{2} \mathrm{O}$ solutions for $30 \mathrm{~s}$ instead of sulfide passivation, which is a general pretreatment for direct bonding of GaAs/ InP pairs that results in highly hydrophobic surfaces.

The bonded GaAs/InP interfaces were analyzed by crack-opening tests and x-ray photoelectron spectroscopy (XPS) through the use of the bonded pairs of semi-insulating GaAs and InP wafers. The crack-opening tests were carried out by inserting $100-\mu \mathrm{m}$-thick razor blades into the bonded interfacial planes and measuring the separation length from IR $(1500 \mathrm{~nm})$ transmission images, and then the bonding strength at the interfaces was determined. ${ }^{6}$ XPS analysis was performed by using $1486.6 \mathrm{eV}$ x-rays generated from an Al $K \alpha$ source, which illuminated the interfacial surfaces of the samples placed into an ultrahigh vacuum immediately after crack opening.

The electrical properties of the bonded $p$-GaAs $/ n$-InP interfaces were measured by using the wafers with carrier concentrations of $8.4 \times 10^{18}$ and $2.8 \times 10^{19} \mathrm{~cm}^{-3}$ for $\mathrm{Zn}$ doped $p$-GaAs and $7.1 \times 10^{18} \mathrm{~cm}^{-3}$ for S-doped $n$-InP, respectively. These pairs will be denoted as $p$-GaAs $/ n^{+}-\mathrm{InP}$ and $p^{+}-\mathrm{GaAs} / n^{+}-\mathrm{InP}$, and their current-voltage $(I-V)$ characteristics were measured with indium metal contacts.

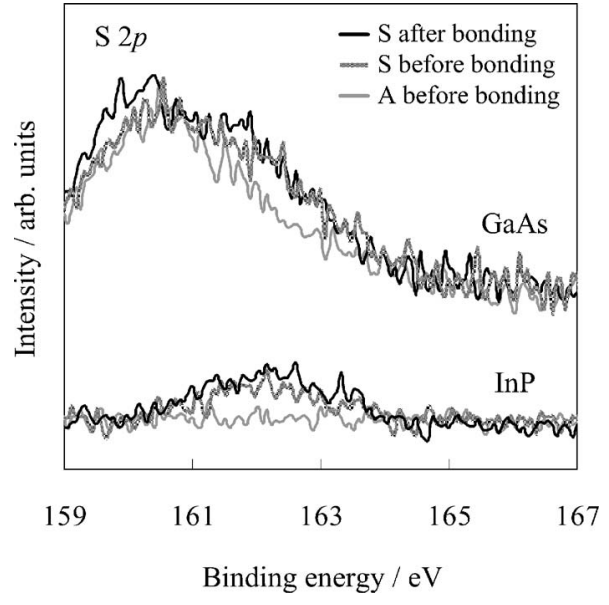

FIG. 2. The XPS spectra of S $2 p$ for sulfide-passivated (S) GaAs and InP surfaces before and after bonding at $300{ }^{\circ} \mathrm{C}$ and for acid-treated (A) GaAs and InP surfaces before bonding.

\section{RESULTS AND DISCUSSION}

\section{A. Chemical and mechanical characterization}

The chemical component at the interfaces of the $\mathrm{A}$ and $\mathrm{S}$ pairs was studied by XPS. Figure 2 shows the XPS S $2 p$ spectra for the interfacial GaAs and InP surfaces. Broad emissions were observed at around $162 \mathrm{eV}$ for both sulfidepassivated wafers (not bonded) and $\mathrm{S}$ pairs bonded at $300{ }^{\circ} \mathrm{C}$ and the feature of the peak was unchanged through the bonding, indicating that the sulfur atoms at the interfaces do not change during the bonding process. However, the remaining sulfur atoms gave no effect on the bonding strength at the interfaces, as shown in Fig. 3. The A and S pairs bonded at temperatures of $200-400{ }^{\circ} \mathrm{C}$ were subjected to the crack-opening tests, resulting in the equal bond strength between the $\mathrm{A}$ and $\mathrm{S}$ pairs, $0.44 \mathrm{~J} \mathrm{~m}^{-2}$ at $200{ }^{\circ} \mathrm{C}$, $0.79 \pm 0.02 \mathrm{~J} \mathrm{~m}^{-2}$ at $300{ }^{\circ} \mathrm{C}$, and $0.96 \pm 0.03 \mathrm{~J} \mathrm{~m}^{-2}$ at $350{ }^{\circ} \mathrm{C}$ in both cases, and the pairs bonded at $400{ }^{\circ} \mathrm{C}$ had the highest bonding strength such that the wafers fractured before crack opened at the bonded interfaces. The weak peak intensities at around $162 \mathrm{eV}$ indicate a small amount of sulfur atoms remaining at the interfaces, which would enable the sulfide-passivated surfaces retain the bonding strength.

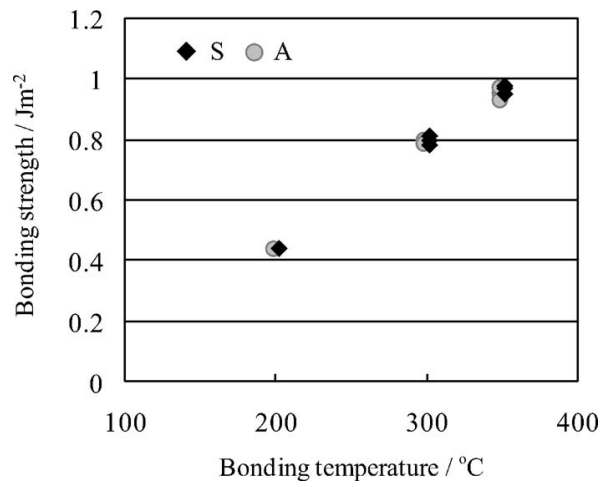

FIG. 3. Bonding strength measured by the crack-open tests as a function of bonding temperature for the sulfide-passivated (S) and acid-treated (A) samples. 
(a)

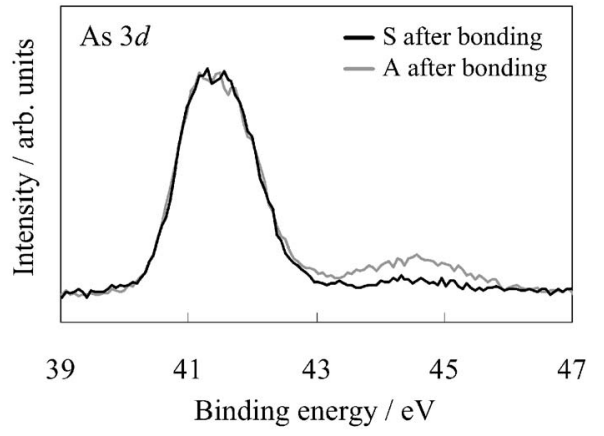

(b)

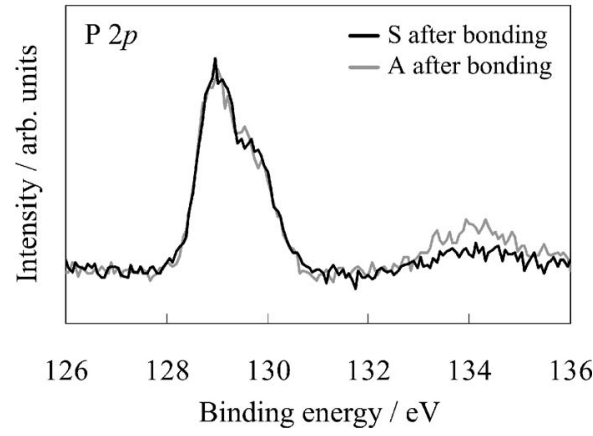

FIG. 4. The XPS spectra of (a) As $3 d$ for bonded GaAs surfaces and (b) $\mathrm{P} 2 p$ for bonded InP surfaces. The black and gray lines correspond to the sulfide-passivated (S) and acid-treated (A) samples, respectively.

The As $3 d$ and $\mathrm{P} 2 p$ spectra for the interfacial surfaces of the $\mathrm{A}$ and $\mathrm{S}$ pairs bonded at $300{ }^{\circ} \mathrm{C}$ are shown in Fig. 4. The large shifted broad emissions at around $45 \mathrm{eV}$ in Fig. 4(a) and around $134 \mathrm{eV}$ in Fig. 4(b), which can be assigned to the oxide species $\left(\mathrm{As}_{2} \mathrm{O}_{3}\right.$ and $\mathrm{As}_{2} \mathrm{O}_{5}$ for $\mathrm{GaAs}$ and $\mathrm{InPO}_{4}$ for InP), were observed for the A pair interfaces, ${ }^{19,20}$ while sulfide passivation led to decreased oxide signals in both the As $3 d$ and $\mathrm{P} 2 p$ spectra. The $\mathrm{O} 1 s$ spectra also revealed that a decreased amount of oxygen atoms exists at the interface of the $S$ pair. These XPS results suggest that the $S$ pairs before bonding, although the surfaces are hydrophilic, involve less oxygen atoms including $\mathrm{H}_{2} \mathrm{O}$ at the interfaces than the hydrophobic A pairs before bonding. The native oxide of the wafers is more effectively etched in the sulfide solution than in the acid solution, leading to a decrease in oxide species at the interface of the $\mathrm{S}$ pairs even after bonding.

\section{B. Electrical characterization}

Figure 5 shows the energy band diagrams for the ideal
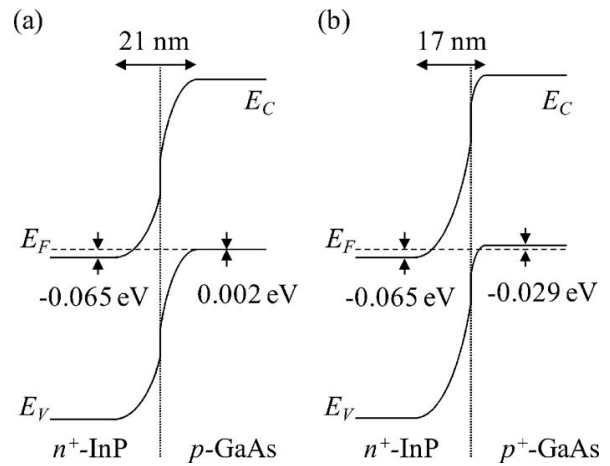

FIG. 5. Ideal energy band diagrams for (a) $p-\mathrm{GaAs} / n^{+}-\mathrm{InP}$ and (b) $p^{+}$-GaAs $/ n^{+}$-InP heterojunctions at thermal equilibrium. The carrier concentrations for $p$-GaAs, $p^{+}$-GaAs, and $n^{+}$-InP are $8.4 \times 10^{18}, 2.8 \times 10^{19}$, and $7.1 \times 10^{18} \mathrm{~cm}^{-3}$, respectively. $E_{C}$ is the energy at the bottom of the conduction band, $E_{V}$ is the energy at the top of the valence band, and $E_{F}$ is the Fermi energy. Both heterojunctions consist of narrow depletion layers with widths of 21 and $17 \mathrm{~nm}$, respectively.

$p$-GaAs $/ n^{+}-\mathrm{InP}$ and $p^{+}-\mathrm{GaAs} / n^{+}-\mathrm{InP}$ heterojunctions used in this study. ${ }^{21}$ The energy band diagrams show monotonically elevated conduction band edges and valence band edges at the interfaces, indicating that the current flow through the junctions would be similar to an ordinal $p$ - $n$ junction, while energy discontinuity in the conduction bands and valence bands leads to the formation of the energy barriers at $n$-GaAs/ $n$-InP and $p$-GaAs/ $p$-InP interfaces. The depletion layer width for both the $p$-GaAs $/ n^{+}-\mathrm{InP}$ and the $p^{+}$-GaAs $/ n^{+}$-InP heterojunctions is considerably narrower than those of conventional $p-n$ junctions, which enables the carriers to tunnel across the layers. However, only the $p^{+}$-GaAs $/ n^{+}$-InP heterojunction fulfills the requirement for carrier tunneling under a forward bias since the Fermi levels are located within the allowed bands on both sides of the junction.

The $I-V$ characteristics of the directly bonded interfaces were measured with the $p$-GaAs sides at a positive bias with respect to the $n$-InP sides. Figures 6(a) and 6(b) show the $I-V$ curves of the $p$-GaAs $/ n^{+}-\mathrm{InP}$ and $p^{+}-\mathrm{GaAs} / n^{+}-\mathrm{InP}$ heterojunctions for the $\mathrm{A}$ and $\mathrm{S}$ pairs bonded at $300{ }^{\circ} \mathrm{C}$ and measured at room temperature. The $\mathrm{S}$ pairs gave a significant improvement in the interfacial conductivity relative to the A pairs under both forward and reverse biases. Here, we emphasize that the $p^{+}-\mathrm{GaAs} / n^{+}-\mathrm{InP}$ heterojunction exhibits the typical behavior of tunnel diodes only when the wafers are sulfide passivated and then bonded [Figs. 6(b) and 6(c)]. This (a)

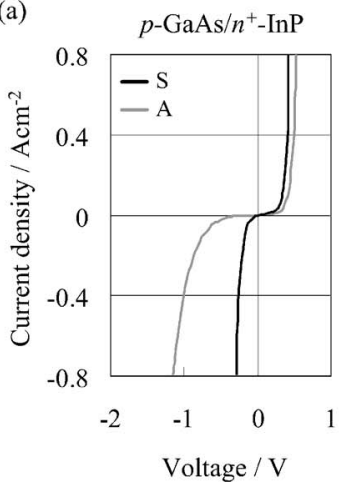

(b)

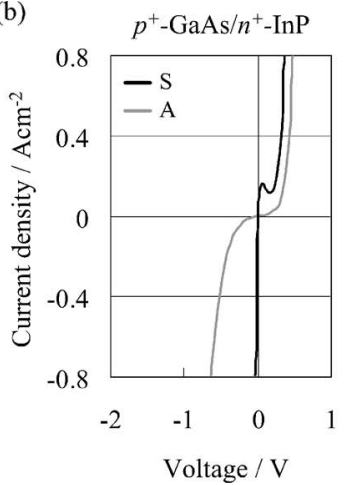

(c)

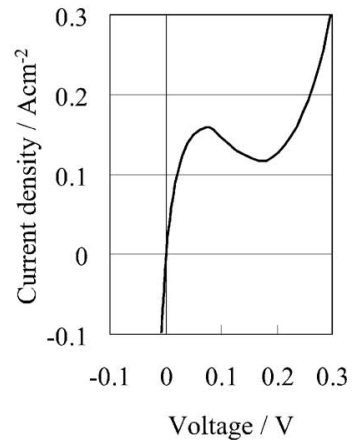

FIG. 6. $I-V$ characteristics for (a) $p$-GaAs $/ n^{+}-\mathrm{InP} \quad$ and $\quad$ (b) $p^{+}-\mathrm{GaAs} / n^{+}-\mathrm{InP} \quad$ heterojunctions bonded at $300{ }^{\circ} \mathrm{C}$. The black lines correspond to the sulfide-passivated (S) and the gray lines correspond to the acid-treated (A) samples. (c) Enlarged $I-V$ curve of the negative resistance region part of (b). 
(a)

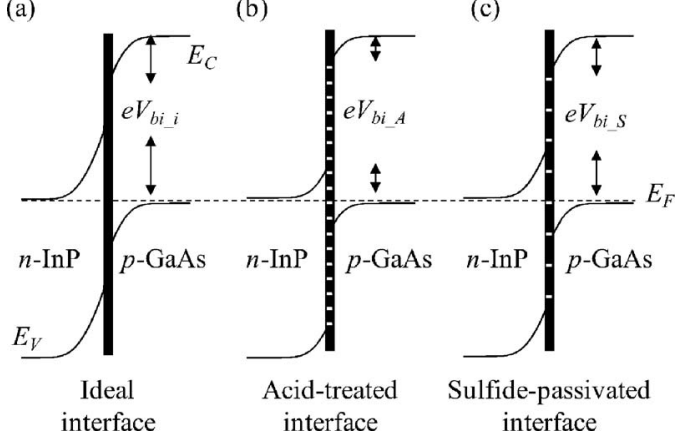

FIG. 7. Energy band diagrams for wafer-bonded $p$-GaAs $/ n$-InP heterojunctions with interfacial trap states (shown by white lines). (a) Ideal interface with no interfacial trap states, (b) acid-treated interface with some interfacial trap states, and (c) sulfide-passivated interface with less interfacial trap states. $V_{\mathrm{bi}}$ represents the total built-in potential of the $p-n$ heterojunction.

sulfide-passivated interface bonded at temperatures as low as $300{ }^{\circ} \mathrm{C}$ showed an Ohmic resistance of $0.13 \Omega \mathrm{cm}^{2}$ in the current region under $0.05 \mathrm{~A} \mathrm{~cm}^{-2}$ and a peak current density of $0.17 \mathrm{~A} \mathrm{~cm}^{-2}$ at the peak voltage of $0.07 \mathrm{~V}$ with a negative resistance, which can be used as the interconnection in multijunction solar cells by considering the operating current density on the order below $20 \mathrm{~mA} \mathrm{~cm}^{-2}$ under 1 sun illumination, although the cells used under concentration have to be interconnected by tunnel junctions with a lower interfacial resistance and a higher peak current density. ${ }^{22}$

The breakdown of the $p-n$ junctions under a reverse bias seen in Fig. 6 is also induced by tunnel effect for the heavy doping concentrations of the wafers used in this study. The $I-V$ curves of the $p$-GaAs $/ n^{+}$-InP heterojunctions for both the $\mathrm{A}$ and the $\mathrm{S}$ pairs under a reverse bias in Fig. 6(a) demonstrate the improved conductivity resulting from sulfide passivation relative to acid treatment, while the $\mathrm{S}$ pair still showed a non-Ohmic $I-V$ characteristic. A slight reverse bias at $p$-GaAs $/ n^{+}-\mathrm{InP}$ should induce an Ohmic increase in the tunnel current because of the increased common band of energies on the $p$-GaAs and $n^{+}$-InP sides seen in Fig. 5(a), implying that ideal heterojunctions did not form after direct wafer bonding with the sulfide passivation in this study.

An ideal $p$-GaAs $/ n$-InP heterojunction at a thermal equilibrium has band bending of both the conduction and the valence band edges with a complete depletion layer and forms a built-in potential $V_{\mathrm{bi} i}$, the sum of the partial built-in potentials at $p$-GaAs and $n$-InP sides [Fig. 7(a)]. However, the bonded $p$-GaAs $/ n$-InP heterojunctions have interfacial trap states resulting from the surface trap states of the wafers, some contaminants involved and misfit defects formed, which traps the carriers at the bonded interfaces in the "depletion" layer. The trapped carriers shield the charge at the interfaces caused by the ionized donors and acceptors, leading to the reduction of band bending, and thus, the built-in potential [Fig. 7(b)].

The effect of sulfide passivation of III-V compound semiconductors on the band bending was extensively studied using Schottky-type structures (i.e., metal semiconductor). ${ }^{16,18}$ The Schottky barrier height on the acidtreated surfaces of both $\mathrm{GaAs}$ and $\mathrm{InP}$ was almost independent of the type of metal, while that on the sulfide-passivated

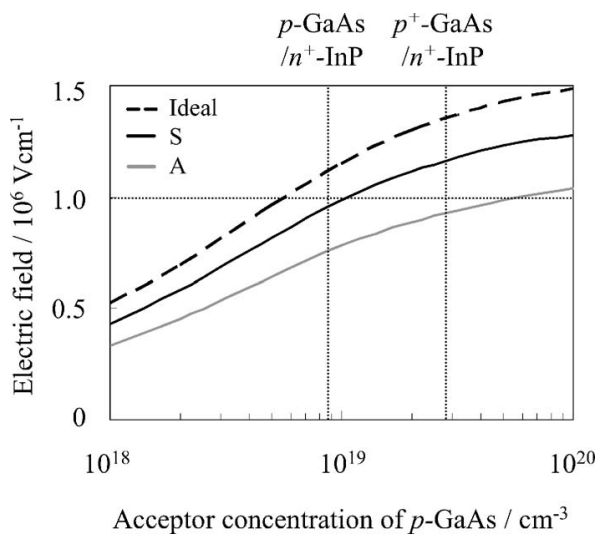

FIG. 8. Calculated electric field at the $p$-GaAs $/ n$-InP interfaces at thermal equilibrium as a function of acceptor concentration of $p$-GaAs with donor concentration of $n$-InP of $7.1 \times 10^{18} \mathrm{~cm}^{-3}$, assuming the reduced built-in potentials at the directly bonded heterojunctions; $V_{\mathrm{bi}_{-} S}=V_{\mathrm{bi}_{-} i}-0.3 \mathrm{~V}$, and $V_{\text {bi_A }}=V_{\text {bi_i } i}-0.6 \mathrm{~V}$.

surfaces varied with the work function of the metal due to the reduction in the surface trap state densities. The effect of sulfide passivation on the band bending of GaAs and InP surfaces can be related to the band bending behavior at the bonded $p-n$ heterojunctions. The reduced interfacial trap state densities at the heterojunction after sulfide passivation would lead to the increase in band bending at the interface so the built-in potential of the $S$ pair $\left(V_{\text {bi_S }}\right)$ is larger than that of the A pair $\left(V_{\text {bi_A }}\right)$ [Figs. 7(b) and 7(c) ]. The higher electric field formed at the interface of the $S$ pair enhances the carrier tunneling across the heterojunctions. Figure 8 shows the calculated electric field at the interface of $p$-GaAs $/ n$-InP heterojunctions at thermal equilibrium as a function of doping concentration of $p$-GaAs. ${ }^{23}$ Ideally, for tunnel current flow, the electric field of both the $p$-GaAs $/ n^{+}-\mathrm{InP}$ and the $p^{+}$-GaAs $/ n^{+}$-InP heterojunctions exceeds $1 \times 10^{6} \mathrm{~V} \mathrm{~cm}^{-1}{ }^{21}$ The electric field at the interfaces of the $\mathrm{A}$ and $\mathrm{S}$ pairs are also calculated assuming that $V_{\mathrm{bi} S}=V_{\mathrm{bi} i}-0.3 \mathrm{~V}$ and $V_{\mathrm{bi} \_\mathrm{A}}$ $=V_{\text {bi } i i}-0.6 \mathrm{~V}$, respectively. This result explains the $I-V$ curves in Figs. 6(a) and 6(b) considering the electric field of $1 \times 10^{6} \mathrm{~V} \mathrm{~cm}^{-1}$ as a threshold value for the tunnel current flow. For the $p^{+}-\mathrm{GaAs} / n^{+}-\mathrm{InP}$ heterojunctions, only the $\mathrm{S}$ pair meets the threshold value allowing tunnel current flow under forward bias. The $\mathrm{S}$ pair with $p-\mathrm{GaAs} / n^{+}-\mathrm{InP}$ and the A pair with $p^{+}$-GaAs $/ n^{+}$-InP allow tunnel current flow after the electric fields reach $1 \times 10^{6} \mathrm{~V} \mathrm{~cm}^{-1}$ and the junctions are broken down under some reverse bias.

\section{CONCLUSION}

Sulfide passivation was employed to reduce the interfacial trap state densities of directly bonded GaAs/InP heterojunctions. XPS measurements confirmed the decrease of oxide species and the presence of sulfur atoms at the bonded interfaces after sulfide passivation. In addition, sulfur atoms at the interface had no effect on the bonding strength. Sulfide-passivated interfaces of the bonded $p$-GaAs $/ n$-InP heterojunctions gave a significant improvement in the conductivity relative to the conventional acid treatment. Tunnel current flow across the interfaces is enhanced under both forward and reverse biases due to the reduction in the inter- 
facial trap state densities caused by sulfide passivation increasing the electric field at the interfaces. Finally, a waferbonded tunnel diode of a heavily doped $p$-GaAs/ $n$-InP heterojunction was successfully fabricated at temperatures as low as $300{ }^{\circ} \mathrm{C}$, which is suitable for the interconnection in a multijunction solar cell since such a low process temperature prevents possible degradation of subcells. To date, we have demonstrated the fabrication of $\mathrm{InGaAs}$ and InGaP/GaAs tandem solar cells on lattice-mismatched $\mathrm{Si}$ substrates via direct wafer bonding and layer transfer. ${ }^{24,25}$ Combining these results with the demonstrated wafer-bonded tunnel diode allows for the fabrication of ultrahigh efficiency latticemismatched multijunction solar cells by directly bonded interconnection between GaAs lattice-matched subcells and InP lattice-matched subcells fabricated on Si substrates.

\section{ACKNOWLEDGMENTS}

This work was supported by the National Renewable Energy Laboratory (NREL). We also acknowledge support from the Center for Science and Engineering of Materials (CSEM), a NSF Materials Research Science and Engineering Center at Caltech, for use of their facilities. Helpful discussions with M. J. Archer is gratefully acknowledged.

${ }^{1}$ A. Marti and G. L. Araujo, Sol. Energy Mater. Sol. Cells 43, 203 (1996). ${ }^{2}$ J. M. Olson, S. R. Kurtz, A. E. Kibbler, and P. Faine, Appl. Phys. Lett. 56, 623 (1990).

${ }^{3}$ M. Yamaguchi, Physica E (Amsterdam) 14, 84 (2002).

${ }^{4}$ J. F. Geisz, S. Kurtz, M. W. Wanlass, J. S. Ward, A. Duda, D. J. Friedman, J. M. Olson, W. E. McMahon, T. E. Moriarty, and J. T. Kiehl, Appl. Phys. Lett. 91, 023502 (2007).

${ }^{5}$ R. R. King, D. C. Law, K. M. Edmondson, C. M. Fetzer, G. S. Kinsey, H. Yoon, R. A. Sherif, and N. H. Karam, Appl. Phys. Lett. 90, 183516 (2007).

${ }^{6}$ Q.-Y. Tong and U. Gösele, Semiconductor Wafer Bonding: Science and
Technology (Wiley, New York, 1998).

${ }^{7}$ P. R. Sharps, M. L. Timmons, J. S. Hills, and J. L. Gray, Proceedings of the 26th IEEE Photovoltaics Specialists Conference (IEEE, New York, 1997), p. 895.

${ }^{8}$ J. M. Zahler, A. Fontcuberta i Morral, C. G. Ahn, H. A. Atwater, M. W. Wanlass, C. Chu, and P. A. Iles, Proceedings of the 29th IEEE Photovoltaic Specialists Conference (IEEE, New York, 2002), p. 1039.

${ }^{9}$ H. Wada, Y. Ogawa, and T. Kamijoh, Appl. Phys. Lett. 62, 738 (1993).

${ }^{10}$ F. Salomonsson, K. Streubel, J. Bentell, M. Hammar, D. Keiper, R. Westphalen, J. Piprek, L. Sagalowicz, A. Rudra, and J. Behrend, J. Appl. Phys. 83, 768 (1998).

${ }^{11}$ K. Tanabe, A. Fontcuberta i Morral, H. A. Atwater, D. J. Aiken, and M. W. Wanlass, Appl. Phys. Lett. 89, 102106 (2006).

${ }^{12}$ R. Stengl, K.-Y. Ahn, T. Mii, W.-S. Yang, and U. Gösele, Jpn. J. Appl. Phys., Part 1 28, 2405 (1989).

${ }^{13}$ C. J. Sandroff, R. N. Nottenburg, J. C. Bischoff, and R. Bhat, Appl. Phys. Lett. 51, 33 (1987).

${ }^{14}$ M. S. Carpenter, M. R. Melloch, M. S. Lundstrom, and S. P. Tobin, Appl. Phys. Lett. 52, 2157 (1988).

${ }^{15}$ H. Sugahara, M. Oshima, H. Oigawa, H. Sigekawa, and Y. Nannichi, J. Appl. Phys. 69, 4349 (1991).

${ }^{16}$ H. Oigawa, J.-F. Fan, Y. Nannichi, H. Sugahara, and M. Oshima, Jpn. J. Appl. Phys., Part 2 30, L322 (1991).

${ }^{17}$ V. N. Bessolov, M. V. Lebedev, and D. R. T. Zahn, J. Appl. Phys. 82, 2640 (1997).

${ }^{18}$ V. N. Bessolov, M. V. Lebedev, A. F. Ivankov, W. Bauhofer, and D. R. T. Zahn, Appl. Surf. Sci. 133, 17 (1998).

${ }^{19}$ M. C. Traub, J. S. Biteen, D. J. Michalak, L. J. Webb, B. S. Brunschwig, and N. S. Lewis, J. Phys. Chem. B 110, 15641 (2006).

${ }^{20}$ A. Nelson, K. Geib, and C. W. Wilmsen, J. Appl. Phys. 54, 4134 (1983).

${ }^{21}$ S. M. Sze and K. K. Ng, Physics of Semiconductor Devices, 3rd ed. (Wiley, New York, 2006).

${ }^{22}$ K. Nishioka, T. Takamoto, T. Agui, M. Kaneiwa, Y. Uraoka, and T. Fuyuki, Jpn. J. Appl. Phys., Part 1 43, 882 (2004).

${ }^{23}$ D. W. Winston, SIMwINDOws Semiconductor Device Simulator, Version 1.5 .

${ }^{24}$ J. M. Zahler, K. Tanabe, C. Ladous, T. Pinnington, F. D. Newman, and H. A. Atwater, Appl. Phys. Lett. 91, 012108 (2007).

${ }^{25}$ M. J. Archer, D. C. Law, S. Mesropian, M. Haddad, C. M. Fetzer, A. C. Ackerman, C. Ladous, R. R. King, and H. A. Atwater, Appl. Phys. Lett. 92, 103503 (2008). 